direction of the beam. In $(a)$ the scattered signal can be seen overlapping the ground signal and extending beyond it. This scatter was found to be strongest when the ground signal was suppressed as in (c), and was itself suppressed nearly at right angles to this direction, as in (b), at the maximum of the ground signal, when the goniometer indicated an almost southerly direction.

(5) The distant scatter group $S$ disappears when the frequency is so high that the ray which leaves the earth tangentially escapes through the $F_{2}$ layer.

These five facts can only be explained in one way. Rays of frequency considerably greater than the critical frequency follow an oblique path, passing through the $E$ layer on their upward path at $A$, and on their downward path at $B$ after reflection at the $F$ layer, as in Fig. 4. Experimental evidence ${ }^{5}$ shows that the $E$ layer is always full of irregular scattering clouds. Signals received at or near the transmitter are those which have been scattered back along their original outward path at $A$, giving rise to echoes of the type $P$, and at $B$, producing the distant scatter group $S$ shown in Fig. 1. The results given in (5) above prove that there are no scattering clouds in the $F$ layer similar to those in the $E$ layer.

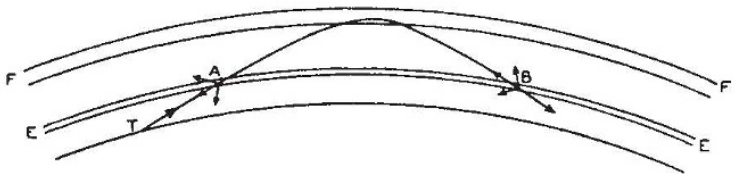

Fig. 4.

All these results are based on very numerous observations carried out over a number of years.

\footnotetext{
${ }^{1} \mathrm{~J}$. Inst. Elect. Eng., "An Investigation of Short Waves", 67, 995-97, (1929).

2 J. Inst. Elect. Eng., "Studies in Radio Transmission", 71, 439-43 (1932).

${ }^{3}$ Marconi Reviewo, "Scattering, Polarisation Errors and Accuracy of S.W. Direction Finding", No. 53 (March-April, 1935) and No. S.W. Direction Finding

4. "Gesammelte Vortrage der Hauptversammlung 1937 der LilienthalGesellschaft für Luftfahrtforschung", pp. 322-329
}

s NATURE, 140, 846 (1937).

\title{
Structure of Proteins
}

$\mathrm{D}^{\mathrm{E}}$ SPITE the cold weather and the proximity of Christmas, some hundred and thirty people attended a meeting of the Physical Society on December 20 at which Dr. Irving Langmuir delivered an address on the structure of proteins. He began by enumerating the facts which pure chemistry had succeeded in accumulating about these substances, which play such an important part in living Nature. By the union of two amino acids, with the elimination of a molecule of water, the polypeptide unit is built up. Chemically, this has two valence bonds free, one at each end, and thus seems adapted solely for the formation of chain compounds. If proteins are indeed chain compounds, it would be difficult to understand why they have apparently definite molecular weights (often multiples of 18,000), or why the molecule behaves approximately as a spherical one.

A fow years ago, Dr. Dorothy Wrinch suggested that, instead of the polypeptide unit (I),

$$
-\stackrel{\mathrm{O}}{\mathrm{C}}-\underset{\mathrm{H}}{\mathrm{C}}-\mathrm{N} / \mathrm{H}
$$

I where $R$ is one of a number of radicals, the unit might really be (II), which differs from it only in the position of a proton within the molecule. It has, however, four free valency bonds, which (being attached to nitrogen or carbon atoms) make angles of $109^{\circ}$ with each other. Investigation of the spatial arrangements possible with such a unit shows that they can form closed 'cages', the plane sides of which are constructed of a lacework of hexagons. The discrete molecular weights (and indeed the ratios of these) thus receive a natural explanation, as does the approximately spherical shape of the molecule.

One cage in particular contains 288 amino-acid residues, which is equal to the number determined by physico-chemical methods in the case of egg albumin, within the limits of accuracy of these methods. In this particular case, the cage is a truncated tetrahedron which does not differ greatly from an octahedron.

Now proteins can be 'denatured' by various mild treatments (shaking for three or four minutes, or heating to $65^{\circ} \mathrm{C}$. for one or two minutes are sufficient in some cases) and then show markedly different properties from native proteins, but no change in molecular weight. It is suggested that these denatured proteins are in fact oomposed of the polypeptides which the chemist had discovered, since the treatment which he necessarily applies in order to make investigations would be sufficient to cause the change. The ease of denaturation, and the great alteration in properties, both follow naturally from this hypothesis, taken in conjunction with the 'cyclol' hypothesis adopted for native proteins.

A particularly interesting fact which receives explanation by these theories is the insolubility in water of mono-molecular layers of proteins formed from soluble native proteins. The explanation lies in the positions of the side chains $R$, which in the cage structure are arranged around the edges of the hexagonal lacunæ composing the plane sides of the cage, whereas in a mono-molecular layer, the cage is developed into a plane. When $R$ is hydrophobe, the mono-molecular layer is formed with these radicals projecting; in the 'cage' they project into the interior and are thus marked.

The last part of the lecture was devoted to the $\mathrm{X}$-ray evidence, which is most complete in the case of insulin, since as shown by Crowfoot, this particular protein has but one molecule per unit cell, and the results are therefore more easily interpreted than would be the case with most proteins.

Dr. Langmuir stressed the point that the X-ray data, like many of the other facts explained by the theory, such as the appearance of $\alpha$-but no $\beta$-amino acids, were not used as data in constructing it originally; nevertheless, the results of the X-ray 
investigation, in Dr. Langmuir's view, confirm many features of the structure to which the theory had led.

In the discussion which followed the address, Dr. Wrinch explained that the method used by Langmuir and Wrinch in interpreting the vector maps was a geometrical formulation of the classical picture of a crystal as a set of discrete units. Prof. J. D. Bernal uttered a word of caution against supposing that the theory was proved. He thought that certain features must be true, and others were possibly true, but in his view the X-ray data fail to confirm the proposed structure. $\mathrm{He}$ had had a vector map calculated for the latter, and found that it differed very markedly from that obtained experimentally. Prof. E. H. Neville showed the care that is needed in interpreting a vector map, by a simple example in which a map constructed from a given set of points appeared at first sight to correspond to quite a different type of distribution.

\section{Studies in Galactic Structure}

$\mathrm{I}^{\mathrm{N}}$ Bulletin of the Astronomical Institutes of the Netherlands, No. 308, J. H. Oort investigates the structure of the galactic system from counts of faint stars in medium and high galactic latitudes combined with absorption data from Hubble's counts of extra-galactic nebulæ. Data for the star density were derived from Kapteyn's Selected Areas; the amount of absorption to be used for each selected area was estimated from nebular counts in neighbouring fields.

Assuming tentatively that the absorption occurs in front of the stars studied, the investigation leads to a picture of the galactic system differing somewhat from the prevalent conception : broadly, the sun appears to be situated in a region of relatively low star density surrounded in all galactic longitudes by extensive regions where the density is at least twice as high. The structural features indicated are large-scale phenomena, extending on both sides of the galactic plane to distances of 500 or 700 parsecs (say 1600 2300 light years) from this plane. The analogy between this structure and the spiral structure of strongly flattened extra-galactic systems is discussed; but the data are insufficient to define the course of the spiral arms, although the sun apparently lies between two arms. At greater distances from the galactic plane, the unevenness in density distribution disappears; at levels between 800 and 1800 parsecs the equidensity surfaces are satisfactorily represented by planes inclined at angles of about $10^{\circ}$ to the galactic plane, implying an increase of density in the direction of the galactic centre by a factor $\mathbf{1} \cdot 38$ per 1000 parsees. The derived longitude of the centre is $324^{\circ} \pm 3^{\circ}$ (region of Scorpio-Sagittarius), in excellent agreement with the longitude derived from the effects of differential rotation or from the distribution of globular clusters and planetary nebulæ. As regards selective absorption, the evidence from colour-excess data in the Kapteyn Areas points to a strong galactic concentration of the absorbing clouds, and indicates that the greater part of the absorption derived from Hubble's counts of nebulæ must have occurred relatively close to the galactic plane.

From the time of Sir William Herschel, the dark patches and lanes, more or less devoid of stars, in the Milky Way have been an attractive problem for astronomers. It is now known that these dark areas must be due chiefly to clouds of cosmic dust. In Publication No. 48 of the Kapteyn Astronomical Laboratory at Groningen, an estimate is made by B. Hiemstra of the distances of some absorption clouds in areas 2, 5, 9 and 24 of Kapteyn's Selected Areas.

From a statistical study of proper motions and apparent magnitudes of the stars in the absorbing regions and in the surrounding rich areas, the author is able to derive limits for the distance and absorption effect of the clouds, assuming them to be of infinitesimal thickness compared with their distance. The results range as follows: Area 5 contains a cloud absorbing $0 \cdot 8^{\mathrm{m}}-1 \cdot 5^{\mathrm{m}}$ at a distance less than 300-400 parsecs. Area 2 contains a cloud absorbing at least $0 \cdot 5^{\mathrm{m}}$ at a distance of $600-1200$ parsecs. In area 9, lying east of the North America Nebula, the absorption reaches $2 \cdot 0^{\mathrm{m}}$ at a distance of 500-650 parsecs. The plates used in the investiga. tion were taken at the Radcliffe Observatory, Oxford, with the 24-inch refractor, which was recently presented to the University of London Observatory at Mill Hill. Usually the pairs of plates were taken about fifteen years apart.

\section{Education for Citizenship}

DREPARATION for and training in citizenship are recognized by an increasing number of people in Great Britain as of vital national importance and as calling urgently for the development and extended application of effective techniques. There is much to interest them in "Forums for Young People", a pamphlet recently issued by the United States Office of Education (Washington, D.C., Superintendent of Documents. 15 cents). This is a study of problems and plans involved in providing organized series of free discussions of public affairs for high school and college students and for other young people. For two years, the Commissioner of Education has conducted, with the help of very substantial Federal grants, a vigorous propaganda for the organization of public affairs forums throughout the States, and the resulting experiments have provided ample material for this study, the publication of which will, he hopes, "contribute to the thinking of the teaching profession and of laymen on this problem of finding ways and means to make secondary and higher education meet its civic responsibilities". It begins with an admirably lucid discussion of pros and cons. The point of view taken is that the most important purpose of education in our time is the training of the mind to deal intelligently with the undecided issues which face mankind. Subsequent chapters describe: types of forum 